\title{
Experimentation and 5G KPI measurements in the 5GENESIS platforms
}

\author{
G. Xilouris ${ }^{1}$, M. Christopoulou ${ }^{1}$, H. Koumaras ${ }^{1}$, M.A Kourtis ${ }^{1}$, M. Emmelmann ${ }^{2}$, D. \\ Trantafyllopoulou, ${ }^{3}$, Y.Rahulan ${ }^{3}$, I. G. Muriel ${ }^{4}$, A. Díaz Zayas ${ }^{4}$, E. Atxutegi ${ }^{5}$, G. Gardikis ${ }^{6}$, D. \\ Lioprasitis $^{6}$, D. Tsolkas ${ }^{7}$, P. Kostakis ${ }^{7}$, E. Aumayr ${ }^{10}$, A.M Bosneag ${ }^{10}$, O. Alay ${ }^{8}$, V. Frascolla ${ }^{9}$, A. \\ Brunström ${ }^{11}$ \\ 1. National Center for Scientific Research "Demokritos", Greece \\ 2. Fraunhofer Institute for Open Communication System FOKUS, Berlin, Germany \\ 3. 5G and 6G Innovation Centres - 5G/6GIC, University of Surrey, UK \\ 4. ITIS Software, Universidad de Málaga, Andalucía Tech, Málaga, Spain \\ 5. Nemergent Solutions SL, Spain \\ 6. Space Hellas (Cyprus) Ltd., Cyprus \\ 7. Fogus Innovations \& Services P.C, Greece \\ 8. University of Oslo \& Simula Metropolitan, Norway \\ 9. Intel Deutschland GmbH, Neubiberg, Germany \\ 10. Ericsson Ireland Research Centre, Ireland \\ 11. Karlstad University, Sweden
}

\begin{abstract}
The adoption of $5 \mathrm{G}$ has recently picked up pace and across the globe commercial deployments are more and more numerous. In addition to better performance, $5 \mathrm{G}$ brings, among others, management and operation flexibility, thus allowing industry verticals to exploit features of the telecommunication networks that go far beyond the new mobile access capabilities. As with any new technology, the integration, testing and validation of new vertical applications pose great challenges at both management and operational levels. In this context, there is an evident need for $5 \mathrm{G}$ infrastructure facilities that offer testing and validation capabilities through a flexible experimentation framework. This paper presents the 5GENESIS EU-funded research project Experimentation Facility and the results of the validation campaigns conducted in its five experimentation platforms. The tests and obtained results were facilitated by the 5GENESIS Suite, an open-source framework providing test automation and result analytics.
\end{abstract}

\section{CCS CONCEPTS}

- Networks $\rightarrow$ Network experimentation; Mobile networks; Network performance analysis.

\section{KEYWORDS}

5G, Network slicing, 5G KPIs, Experimentation, 5G Trials

\section{INTRODUCTION}

The recently emerged fifth generation $(5 \mathrm{G})$ of cellular communication provides support for heterogeneous services with a very high level of flexibility. The versatility of the $5 \mathrm{G}$ technology allows an effective and scalable support to the current, and also potentially forthcoming, diverse enhancements and functionalities required by industry verticals. In addition, advances and capabilities, such as network softwarisation and virtualization, are adopted and wellintegrated into the $5 \mathrm{G}$ system via new concepts such as network slicing [13]. This $5 \mathrm{G}$ feature targets at exploiting a single infrastructure for facilitating multiple verticals' requirements and diverse Key Performance Indicators (KPIs) exploiting a single infrastructure to fulfill all requirements. Network slices are expected to be operating concurrently, presenting substantial challenges to the $5 \mathrm{G}$ commercial deployments [10].

In addition to the previous challenges centered around the specifications of the $5 \mathrm{G}$ environment, challenges also exist for the facilitation of vertical sectors' services design and integration. In order to both ease the adoption of 5G technology and provide experimenters with a flexible and easy to use means to validate 5G KPIs, there is a growing demand for open and accessible $5 \mathrm{G}$ experimentation facilities. These facilities should allow access to experimenters, developers and testing teams to develop, deploy, test and validate new services as well as running services in a real testing $5 \mathrm{G}$ environment. As a response to this demand, the European Commission funded three projects for creating such end-to-end (E2E) 5G infrastructures, namely 5GENESIS [12], 5G-VINNI [14], and 5G-EVE [11]. The value proposition of 5GENESIS is therefore to address the above mentioned demand for open facilities, making available to experimenters a well-articulated, open and flexible experimentation framework and a set of tools tuned to ease the execution and monitoring of experiments.

This paper provides a summary of the evolution of the architecture proposed by 5GENESIS, which is composed of five platforms, i.e., Malaga, Athens, Limassol, Surrey and Berlin, and main results from the first (out of the three planned) experimentation campaigns, collected across the participating platforms. The main contribution of the paper is to present and discuss the acquired experimental results for a number of KPIs and vertical use case deployments [6]. The results were all acquired via the automated 5GENESIS experimentation framework, based on pre-commercial and open $5 \mathrm{G}$ 


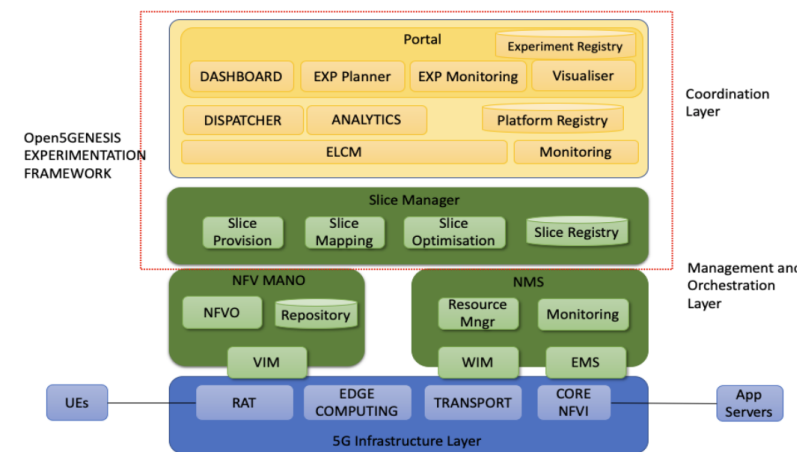

Figure 1: Open 5GENESIS Suite Architecture

components, and serve as a guide for the second experimentation phase based of new integration activities and more mature, closer to market, technologies. In this context the second phase will also provide KPI validation and highlight the deltas and the evolution that is achieved.

The paper is structured as follows. Section II provides a brief description of the 5GENESIS experimentation framework. Section III discusses the available 5GENESIS platforms and respective setups. Section IV provides the experimental results, and Section V concludes the paper.

\section{5G EXPERIMENTATION FRAMEWORK}

The overall 5GENESIS architecture that facilitates $5 \mathrm{G}$ experimentation is illustrated in Fig. 1. From bottom up, the architecture is divided in three layers, namely the Infrastructure Layer, provided by the five 5GENESIS platforms, the Management and Orchestration (MANO) Layer, and the Coordination Layer. The MANO Layer is responsible for the management and orchestration aspects of each platform and comprises of infrastructure components which may vary for each platform i.e., the Element Management Systems (EMS), the WAN Infrastructure Manager (WIM), the Virtualization Infrastructure Managers (VIMs), the NFV Orchestrator, and the Slice Manager. The Coordination Layer includes all the necessary components for instantiating and managing an experiment, as well as the facility's monitoring and telemetry components. The components of the Coordination Layer and the Slice Manager of the MANO Layer are provided by the 5GENESIS Experimentation Suite [5] (see red rectangle in Fig. 1).

The 5GENESIS Suite is a software suite spanning across the Coordination Layer and the Management and Orchestration Layer. 5GENESIS provides all experiment related actions such as planning, execution and data analytics, handles the interfacing with the infrastructure, and manages the interfacing with the experimenters. From top to bottom, the Portal module provides a Graphical User Interface (GUI) for designing and planning of experiments, supervising target metrics while the experiment is executed, visualizing of results, as well as accessing the experiment's analytics. The Experiment Lifecycle Manager (ELCM) controls all experiment's lifecycle events, e.g., setup, start, pause, stop, etc. It can also configure, schedule and compose multiple experiments that need to run in sequence or concurrently. The link between the Portal and the ELCM is formed by the Dispatcher, which is the component that provides all the OpenAPIs, exposing internal components functionalities, as well as APIs acting as mediators imposing additional access security.

The 5GENESIS Analytics module provides methods to analyze the recorded data from experiments that have been carried out in 5GENESIS platforms, enabling the experimenter to validate the desired KPIs. It uses a service-based architecture, where different analytical methods are provided as individually scalable services. The analytical methods comprise time series overview, statistical validation, correlation analysis and predictive analysis services, along with data retrieval and pre-processing, including time series synchronization and outliers removal.

The 5GENESIS Slice Manager [4] is an open-source software component developed within the project, responsible for controlling all infrastructure elements involved in the realization of the slice, as well as providing a Northbound Interface (NBI) for creating, modifying, monitoring and deleting slices. Through the NBI, Katana interacts with a coordination layer or directly with the administrator of the Facility. It receives the Network Slice Template (NEST) to create network slices and provides the API for managing and monitoring them.

Each infrastructure domain, i.e., radio, network, core and edge cloud, is treated separately in order to provide the proper E2E mapping, thus facilitating the creation of different Service Slice Types (i.e., eMBB, URLLC, and mMTC) or variations depending on the experimenter's requirement.

The experimenter can employ the provided analytical methods, such as time series-, statistical-, and correlation-analysis, to easily inspect the behavior and relations between KPIs to ensure a correct functionality of the experiment setup. Any unexpected behavior, e.g., sudden and intermittent drops in throughput or a correlation between KPIs that should not occur, can indicate a malfunction in the setup that should immediately be remedied. 5GENESIS defines a series of KPI test cases in the form of executable scripts that automate network slice provision, component deployment, data collection agents and traffic probes as well as any additional configuration needed. A detailed description of the proposed methodology is provided in [8].

\section{5G EXPERIMENTATION PLATFORMS}

5GENESIS consists of five experimental platforms located at Malaga, Athens, Limassol, Surrey, and Berlin. Detailed deployment and functional information is available at the respective D4.xx Deliverables [3]. These platforms operate as standalone experimentation facilities, each one implementing a variation of the 5GENESIS reference architecture adapted to the demonstration scenarios and KPI validations each platform can support. The 5GENESIS Suite is deployed in all platforms and is well integrated with the infrastructure components. The benefits of using 5GENESIS Suite for the KPI validation and experiments is due to the automation of experiment execution, analytics and result presentation. Hence the 5GENESIS Suite achieves measurement process repeatability and comparability under all scenarios and equipment used.

The Málaga Platform is oriented to validate KPIs for verticals related to Mission Critical Services (MCS), which require Enhanced 
Table 1: 5GENESIS Platforms Radio Configuration

\begin{tabular}{|c|c|c|c|c|c|c|}
\hline $\begin{array}{l}\text { Deployment } \\
\text { Parameters }\end{array}$ & \multicolumn{2}{|c|}{ Athens } & Malaga & Limassol & \multirow{2}{*}{$\begin{array}{l}\text { Surrey } \\
\text { 5GIC Rel.15 4G } \\
\text { Core }\end{array}$} & \multirow{2}{*}{$\begin{array}{l}\text { Berlin } \\
\text { Open5GCore } \\
\text { Rel.5 }\end{array}$} \\
\hline Core Network & $\begin{array}{l}\text { Amarisoft EPC } \\
\text { Rel.15 (Athens } \\
\text { 1) }\end{array}$ & $\begin{array}{l}\text { Athonet EPC } \\
\text { Rel.15 (Athens } \\
\text { 2) }\end{array}$ & $\begin{array}{l}\text { Athonet EPC } \\
\text { Rel.15 }\end{array}$ & $\begin{array}{l}\text { Amarisoft EPC } \\
\text { Rel. } 15\end{array}$ & & \\
\hline 3GPP Mode & \multicolumn{2}{|l|}{ NSA Option 3a } & NSA Option $3 \mathrm{x}$ & NSA Option 3 & NSA Option 3 & SA \\
\hline Frequency & \multicolumn{2}{|c|}{ 4G: $2.1 \mathrm{GHz}, 5 \mathrm{G}: 3.5 \mathrm{GHz}$} & $\begin{array}{l}\text { 4G: } 2.6 \mathrm{GHz}, 5 \mathrm{G}: \\
3.5 \mathrm{GHz}\end{array}$ & $\begin{array}{l}\text { 4G: } 2.1 \mathrm{GHz}, 5 \mathrm{G}: \\
3.5 \mathrm{GHz}\end{array}$ & $\begin{array}{l}\text { 4G: } \quad 700 \mathrm{MHz}, \\
2.6 \mathrm{GHz}, 5 \mathrm{G}: 3.5 \\
\mathrm{GHz}\end{array}$ & 5G: $3.7 \mathrm{GHz}$ \\
\hline Bandwidth & \multicolumn{2}{|c|}{ 4G: $10 \mathrm{MHz}, 5 \mathrm{G}: 50 \mathrm{MHz}$} & $\begin{array}{l}\text { 4G: } 20 \mathrm{MHz}, 5 \mathrm{G}: \\
40 \mathrm{MHz}\end{array}$ & $\begin{array}{l}\text { 4G: } 10 \mathrm{MHz} \\
\text { 5G: } 50 \mathrm{MHz}\end{array}$ & $\begin{array}{l}\text { 4G: } 20 \mathrm{MHz}, 5 \mathrm{G}: \\
100 \mathrm{MHz}\end{array}$ & 5G: $100 \mathrm{MHz}$ \\
\hline MIMO Layers & \multicolumn{2}{|c|}{ 4G: MIMO 2x2 5G: MIMO 2x2 } & $\begin{array}{l}\text { 4G: MIMO } 4 \times 4 \text {, } \\
\text { 5G: MIMO } 2 \times 2\end{array}$ & $\begin{array}{l}\text { 4G: MIMO 2x2, } \\
\text { 5G: MIMO 2x2 }\end{array}$ & $\begin{array}{l}\text { 4G: Omni } \\
\text { 5G: MIMO 64x64 }\end{array}$ & 5G: MIMO 2x2 \\
\hline Duplex Mode & \multicolumn{2}{|c|}{ 4G: FDD, 5G: TDD } & $\begin{array}{l}\text { 4G: FDD } \\
\text { 5G: TDD }\end{array}$ & $\begin{array}{l}\text { 4G: FDD } \\
\text { 5G: TDD }\end{array}$ & $\begin{array}{l}\text { 4G: FDD \& TDD } \\
\text { 5G: TDD }\end{array}$ & 5G: TDD \\
\hline $\begin{array}{l}\text { TDD } \\
\text { DL:UL ratio }\end{array}$ & \multicolumn{2}{|l|}{$7: 2$} & $8: 2$ & $7: 2$ & $8: 2$ & $8: 2$ \\
\hline UE & \multicolumn{2}{|l|}{ Samsung A90 5G } & $\begin{array}{l}\text { Samsung Galaxy } \\
\text { Note } 105 \mathrm{G}\end{array}$ & Samsung A90 5G & $\begin{array}{l}\text { Huawei } 5 \mathrm{G} \mathrm{CPE} \\
\text { Pro }\end{array}$ & $\begin{array}{l}\text { Huawei P40 } \\
\text { Huawei CPE 5G } \\
\text { Pro \& Pro2 }\end{array}$ \\
\hline Chipset & \multicolumn{2}{|c|}{ Qualcomm SM8150 Snapdragon 855} & Exynos 9825 & $\begin{array}{l}\text { Qualcomm } \\
\text { SM8150 Snap- } \\
\text { dragon } 855\end{array}$ & $\begin{array}{l}\text { Balong } 5000 \\
\text { multi-mode }\end{array}$ & $\begin{array}{l}\text { Kirin } 9905 \mathrm{G} \\
\text { Balong } 5000 \\
\text { multi-mode }\end{array}$ \\
\hline
\end{tabular}

Mobile Broadband and Low Latency Communications. The platform combines an indoor and outdoor $4 \mathrm{G}$ and $5 \mathrm{G}$ deployment at the campus of the University of Málaga, a $4 \mathrm{G}$ and $5 \mathrm{G}$ outdoor deployment in the city centre of Málaga, along with the support of commercial Mobile Network Operators (MNOs), core providers and MCS services providers. Malaga platform is an evolution of the previous $4 \mathrm{G}$ platform TRIANGLE [16].

The Athens Platform comprises of three sites located in Athens city extended area, namely the National Center for Scientific Research "Demokritos", the OTE Academy campus and the Egaleo Stadium. The sites are interconnected via wireless and wired links in order to form an expanded $5 \mathrm{G}$ infrastructure integrating a series of radio access technologies (RATs), and vertical use cases. The supported verticals include $5 \mathrm{G}$ Controlled Drone for surveillance, big events media coverage, and Security as a Service (SECaaS) applications.

The Limassol Platform integrates several infrastructures in the city of Limassol, Cyprus, in order to form an interoperable multiradio facility, combining terrestrial and satellite communications with the ultimate aim of efficiently extending $5 \mathrm{G}$ coverage to underserved areas. To that end, the Limassol $5 \mathrm{G}$ platform employs NFV/SDN-enabled satellite communications as well as tight integration of different access and backhaul technologies.

The Surrey Platform is hosted in the 5G Innovation Centre (5GIC) at the University of Surrey, UK. In 5GENESIS, the Surrey platform is used to demonstrate the capability of $5 \mathrm{G}$ networks to use and exploit multiple RATs, both 3GPP and non-3GPP, that can support mMTC, and multimedia services. The platform supports commercial 4G and 5G New Radio (NR) and features such as network slicing, function virtualization, SDN control and mobile edge computing. Both stand-alone (SA) and non-standalone (NSA) network configurations are supported, providing coverage based on commercial LTE and 5G NR operating in 700, 2600, and $3500 \mathrm{MHz}$ bands.

The Berlin Platform centres around the 5G-Playground testbed, which is extended within 5GENESIS with a multi-vendor 5G SA NR UEs and gNBs connected to the Open5GCore [9] and an underlying 100G-based, application-centric network and virtualization infrastructure. Apart from conducting experiments at the Fraunhofer FOKUS platform, it allows for temporal field test in the Berlin city centre at Humboldt University campus used for field-trials, as well for dedicated evaluations of $60 \mathrm{GHz}$ NR equipment located at IHP, Frankfurt Oder.

\section{VALIDATION OF NETWORK KPIS}

This section presents the network KPI validation results. Although not all tests have been executed in all platforms, all tests followed the same methodology as presented in [8], by using the same procedure, ancillary tools and analytics. According to the methodology, each KPI validation experiment is defined by a test case file describing the measurement approach, the primary and complementary metrics as well as the statistical analysis to obtain the main KPI results. All the experiments are associated with a specific scenario 
Table 2: Platform Throughput Measurements

\begin{tabular}{|l|c|c|}
\hline Setup ID & $\begin{array}{c}\text { Average } \\
\text { UDP Throughput } \\
\text { (Mbps) }\end{array}$ & $\begin{array}{c}\text { Average } \\
\text { TCP Throughput } \\
\text { (Mbps) }\end{array}$ \\
\hline Athens_1 & 369.27 & - \\
\hline Athens_2 & 363.27 & - \\
\hline Malaga & 264.74 & - \\
\hline Limassol & - & 212.72 \\
\hline Surrey & - & 492.08 \\
\hline Berlin & - & 115.32 \\
\hline
\end{tabular}

Table 3: RTT Measurements across 5GENESIS Facilities

\begin{tabular}{|l|c|c|c|}
\hline Setup & Mean [ms] & Min [ms] & Max [ms] \\
\hline Athens_1 & 34.66 & 21.6 & 51.18 \\
\hline Athens_2 & 32.35 & 19.88 & 48.25 \\
\hline Malaga & 12.45 & 10.02 & 20.78 \\
\hline Limassol & 32.26 & 17.32 & 47.42 \\
\hline Surrey & 10.47 & 8.23 & 15.6 \\
\hline Berlin & 11.3 & 9.8 & 12.6 \\
\hline
\end{tabular}

used for the testbed setup (i.e ideal UE reception etc). It should be noted that the presented results are not directly comparable among all platforms, due to the pre-commercial equipment used and the deployment setup and configuration of each platform.

\subsection{Throughput}

Throughput adheres to the data (payload) successfully transferred within a given time period from a data source to a data sink [7]. It depends on the radio conditions, the configuration of the gNB, such as the maximum achievable modulation scheme, MIMO configuration, available bandwidth, duplex mode and TDD configuration.

5GENESIS includes test cases for evaluating both UDP and TCP throughput. The former provides the maximum (peak) achievable throughput without taking into consideration the correct reception of the transmitted information. On the other hand, the latter establishes the connection via the three-way handshake and provides error recovery at the expense of lower speed. Athens and Malaga selected the UDP tests in order to measure the peak data rate of the $5 \mathrm{G}$ link.

Table 2 provides the results of the throughput experiments. These measurements correspond to different radio and core configurations in each platform, as shown in Table 1. It must be noted that all tests described in this paper have been defined, scheduled, automatically executed and processed leveraging the capabilities of the Open5GENESIS suite.

In the Berlin Platform, the throughput experienced over initial 5G SA set-up highly varied depending on the used combination of $\mathrm{UE} / \mathrm{gNB}$ manufacturer and firmware versions. Measurements were based on non-final, pre-commercial firmware releases.

\subsection{Round Trip Time (RTT)}

RTT represents the time it takes to transfer a given piece of data between two nodes, to process it at the receiving node, and to transfer

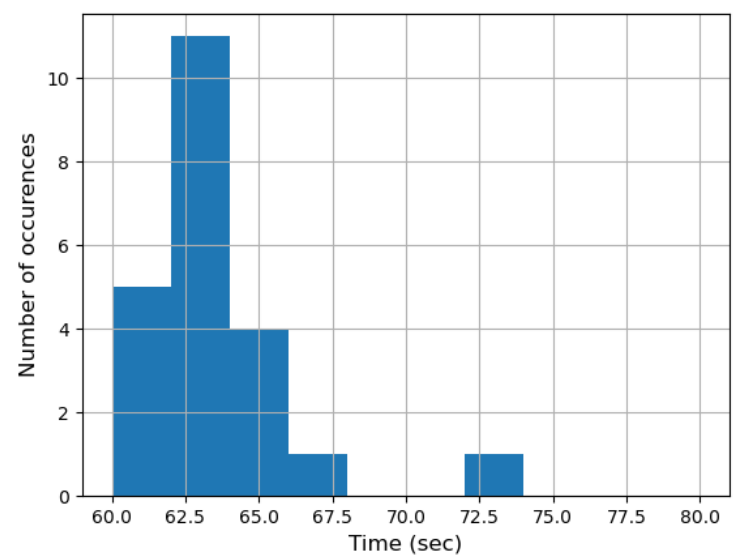

Figure 2: Service Deployment Time measured at the Athens 5GENESIS Platform.

an acknowledgement status back to the transmitting node, measured from the moment the info is transmitted by the application to the moment the acknowledgement status is received [7].

These experiments evaluated RTT between commercial 5G UE and an Application Server. The measurements were conducted using the ICMP protocol and while the Application Server was connected at N6 or SGi interface with the $5 \mathrm{G}$ core operating in SA or NSA mode respectively. Table 3 provides the detailed results per setup, including the mean, minimum and maximum RTT values when transmitting packet sizes of 64 bytes. Differences in the experienced RTTs are caused by using different UEs, gNB and firmware combinations, different vendors of the $5 \mathrm{G}$ core, and platform-specific performance of the underlying network components as well as compute and storage performance of the virtualization infrastructure.

\subsection{Service Deployment Time}

The 5GENESIS framework integrates the Slice Manager placed between the Experiment Coordination Layer and the Orchestration Layer, as discussed in the previous section. The provision of resources for the creation of a network slice and the instantiation of all the vertical application components is a pre-requisite for the initiation of an E2E test case. A KPI for the assessment of the duration of the provision of resources across all technology domain and the creation of a Network Slice including vertical application components deployment and physical and virtual function configuration is the Service Deployment Time (SDT). The aforementioned KPI is actually a sum of the duration of a great number of procedures and interactions that take place since the arrival of a service deployment request till the fulfillment of this request, making an E2E service available to the End Users.

As suggested in [2] several factors affect the SDT. The service instantiation process includes several steps until full deployment and service activation.Those steps are:

- Parse slice deployment request 


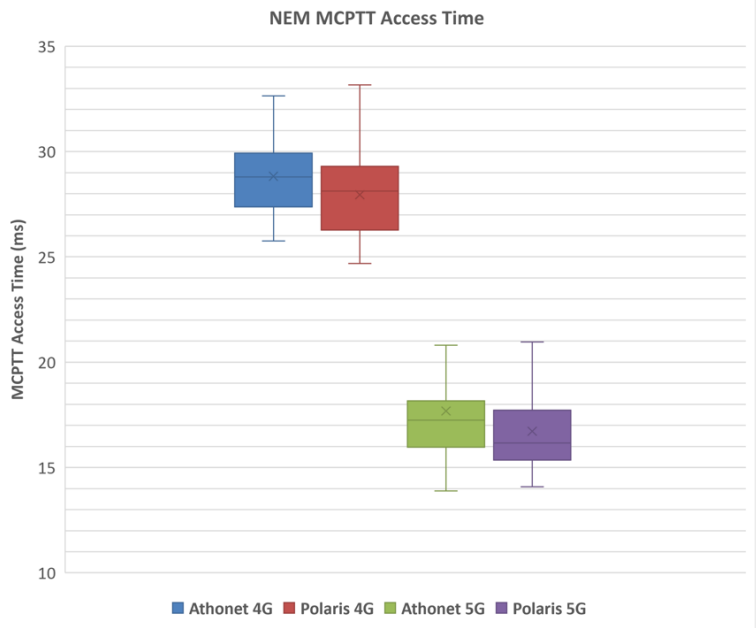

(a)

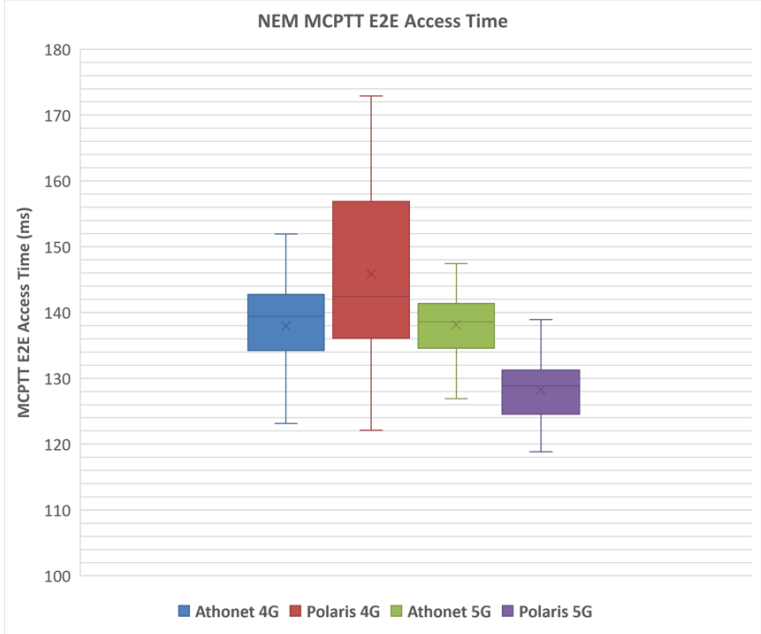

(b)

Figure 3: MCPTT results with two 4G and 5G cores: a) MCPTT Access Time; b) MCPTT E2E Access Time

- Calculate placement and resource availability

- Resource provisioning by communicating with Orchestrator (NFVO), VIMs and Network Managers (NMS)

- VNF on-boarding on VIMs and instatiation

- Network elements (WAN, Radio) configuration by NMS

- Service activation

The first step is the parsing of the request in order to identify the required resources and placement requirements for each VNF and vertical application component. Then when the placement has concluded, the resources are provisioned through a series of interactions between the Slice Manager and the Orchestrator (NFVO), the Virtualization Infrastructure Managers (VIM) and Network Manager (NMS). Following this step, the NFVO is instructed to on-board the VNFs and instantiate them at the designated locations, utilizing the available provisioned resources. In sequence the NMS is instructed to configure all network elements (WAN, Radio) to successfully provide users with internet connectivity. As a final step the service is activated. From the above it is obvious that the SDT is dependent on various factors [2].

The deployed service includes a 5 G connectivity Network Service comprising of a virtual EPC (vEPC) and a SD-RAN based gNB (Amarisoft) as well as a dummy VNF representing the vertical application. The backhaul is implemented via a WAN infrastructure that supports SDN. All the deployments and configurations that take place are automated.

The experiment measures the duration of the process, beginning from the moment the request is received by the Slice Manager, until the Mobile Network is fully operational and ready to accept UE's connections. The Slice Creation Time Records are reported for each one of the 25 Service Creation requests individually and the result is averaged over these iterations. Figure 2 illustrates the histogram of the Service Creation Times measured. It can be seen that the service deployment time in all executions is below $73 \mathrm{~ms}$, with an average of $64 \mathrm{~ms}$.

\section{VERTICAL EXPERIMENTATION}

\subsection{Mission Critical Services}

3GPP-compliant MCS are part of use cases related to the 5GENESIS Málaga Platform. In this regard, different experiments were conducted in order to measure the KPIs related with Mission Critical Push-to-Talk (MCPTT). The metrics measured have been MCPTT Access Time and MCPTT E2E Access Time. The former is defined as the time elapsed from the moment the user requests to speak to the moment permission to speak is granted during an ongoing MCPTT call without involving confirmation of any other user. The latter consists of a similar procedure but this time requiring other user confirmation in the MCPTT call establishment.

The mentioned standardized KPIs [1] have been measured for both $4 \mathrm{G}$ and $5 \mathrm{G}$ NSA, in order to compare and confirm the improvement in 5G, gathering the required signaling in lower layers of the UEs to avoid as much as possible UE-specific delays. In addition, for those two scenarios the metrics have been measured with two different vendor cores (i.e., Polaris and Athonet). The results of the experiments have shown an improvement, approximately of $10 \mathrm{~ms}$ and about $13 \mathrm{~ms}$ in MCPTT Access Time and MCPTT E2E Access Time respectively, when using $5 \mathrm{G}$ NSA in comparison with $4 \mathrm{G}$, as can be seen in Figure 3. It is important to highlight that MCPTT E2E Access Time has shown greater dependency with the core progress towards $5 \mathrm{G}$, demonstrating similar results as for $4 \mathrm{G}$ for one core and the above mentioned improvement for the other one. The results primarily show the positive impact that the evolved technology components could bring to verticals by providing a more convenient field to reach standard 3GPP-defined KPI thresholds, that will result in better QoS and QoE for first-responders. Ensuring fast and reliable service is key for them and the results show a great improvement that could benefit their service speed in daily operations and therefore responsiveness. 


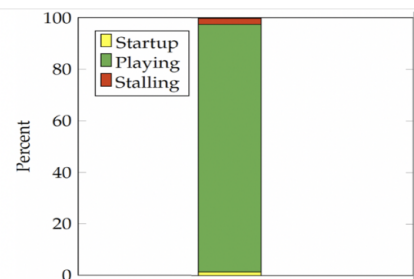

(a)

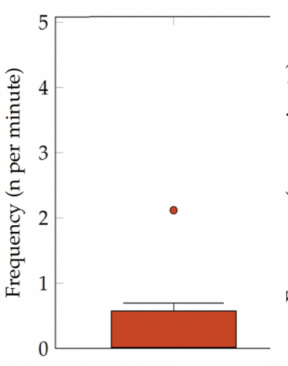

(c)

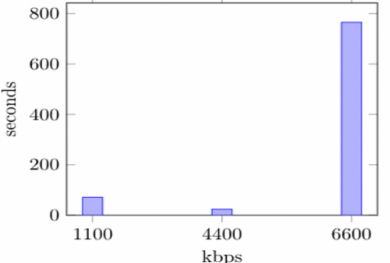

(b)

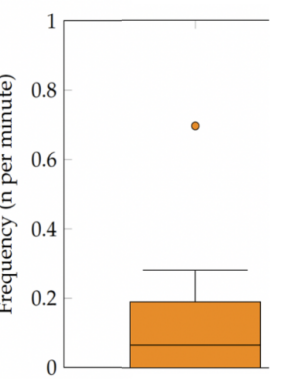

(d)

Figure 4: 360-degree Video Experience in Festival of Lights: (a) average times spent on different player states, (b) total times spent playing on different bit rate levels, (c) rebuffering event frequency and d) quality switch.

\subsection{0-degree Video Streaming}

The main use case for 5GENESIS Berlin platform is media and video distribution in a dense urban environment. To this end, we deployed a 360-degree video system running [15] over a 5G SA network core at the courtyard of Humboldt University (HU). The edge deployment was located in a car (van) that features a commercial, data-center-graded compute and storage infrastructure (NetApp HCI system), which was used to host the Open5GCore and the video processing software.

The video camera, producing a 360-degree view of the illuminations at this festival, was connected to the Open5GCore using the provided UE/gNB emulation as 5G SA RAN equipment was not available during the field trials conducted in 2019. Test users could access the system via a deployed Wi-Fi access network. The edge was connected via a $60 \mathrm{GHz}$ backhaul to the GÉANT network towards the main datacenter at the FOKUS site, which allowed for accessing the video data processed in the edge from a (public) Internet access.

The goal of this first trial was to gain experience with the nomadic, edge-based deployment at $\mathrm{HU}$ and to obtain first experiment results. In our trials, we have evaluated 360-degree video streaming performance in terms four different criteria as illustrated in Fig 5. Overall, we observe short start up times and very few re-buffering events. The video quality has also been adapted to use the highest available quality with very few switches back to the lower quality. This illustrates that the current configuration can handle the demanding 360-degree video streaming applications.

\section{CONCLUSIONS}

This work presents experimental results conducted by the 5GENESIS platforms, acquired through the Open5GENESIS Suite. The latter provides a testing and experimentation framework capable of automating the experimentation, collection and analysis process over a real $5 \mathrm{G}$ infrastructure. The 5GENESIS platform gives the capability to experimenters to design test cases and scenarios and obtain analysed and raw results. The experiment repeatability provides the capability to execute multiple experiments with a variety of testbed configurations and comparability between the obtained experiment results. The platform automates the experimentation process and at the same time integrates infrastructure components at management level in order to orchestrate the creation of network slices used for conducting the experiments. The difference between same experiments on different platforms demonstrate the significant impact the different infrastructure deployments have on the results. The 5GENESIS Suite as well as the 5GENESIS platforms are continuously evolving, and a new phase of tests with more stable components and optimised configurations is planned.

\section{ACKNOWLEDGEMENTS}

This work has been conducted under the auspices of the 5GENESIS project under grant agreement No 815178.

\section{REFERENCES}

[1] 3GPP. 2019. SMission Critical Push to Talk (MCPTT); Stage 1. Technical Specification (TS). 3rd Generation Partnership Project (3GPP).

[2] Idris Badmus, Abdelquoddouss Laghrissi, Marja Matinmikko-Blue, and Ari Pouttu. 2020. Identifying Requirements Affecting Latency in a Softwarized Network for Future 5G and Beyond. In 2020 2nd 6G Wireless Summit (6G SUMMIT). 1-6. https://doi.org/10.1109/6GSUMMIT49458.2020.9083785

[3] 5GENESIS Consortium. [n.d.]. 5GENESIS Platform Deliverables D4.xx. https: //5genesis.eu/deliverables Accessed: February 2021.

[4] 5GENESIS Consortium. [n.d.]. KATANA 5G Slice Manager. https://bit.ly/ 37yH84O Accessed: February 2021.

[5] 5GENESIS Consortium. [n.d.]. Open5GENESIS Suite. https://github.com/ 5genesis. Accessed: February 2021.

[6] 5GENESIS Consortium. 2020. Deliverable 6.2:Trials and experimentation (cycle 2). Technical Report. https://5genesis.eu/wp-content/uploads/2020/08/5GENESIS_ D6.2_v1.0_FINAL.pdf

[7] 5GENESIS Consortium. 2020. Trials and experimentation (cycle 1). Technical Report. http://5genesis.eu/wp-content/uploads/2019/12/5GENESIS_D6.1_v2.00. pdf

[8] Almudena Díaz-Zayas, Giuseppe Caso, Özgü Alay, Pedro Merino, Anna Brunström, Dimitris Tsolkas, and Harilaos Koumaras. 2020. A Modular Experimentation Methodology for 5G Deployments: The 5GENESIS Approach. Sensors 20, 22 (2020), 6652. https://doi.org/10.3390/s20226652

[9] Fokus. [n.d.]. Open5GCore. https://www.open5gcore.org, Accessed: February 2021.

[10] Peng Guan, Dan Wu, Tingjian Tian, Jianwei Zhou, Xi Zhang, Liang Gu, Anass Benjebbour, Masashi Iwabuchi, and Yoshihisa Kishiyama. 2017. 5G Field Trials: OFDM-Based Waveforms and Mixed Numerologies. IEEE fournal on Selected Areas in Communications 35, 6 (2017), 1234-1243. https://doi.org/10.1109/JSAC. 2017.2687718

[11] Milon Gupta, Rodolphe Legouable, Marc Molla Rosello, Maurizio Cecchi, Jaime Ruiz Alonso, Manuel Lorenzo, Evangelos Kosmatos, Mauro R. Boldi, and Gino Carrozzo. 2019. The 5G EVE End-to-End 5G Facility for Extensive Trials. In 2019 IEEE International Conference on Communications Workshops (ICC Workshops). 1-5. https://doi.org/10.1109/ICCW.2019.8757139

[12] Harilaos Koumaras, Dimitris Tsolkas, Georgios Gardikis, Pedro Merino Gomez, Valerio Frascolla, Dionysia Triantafyllopoulou, Marc Emmelmann, Vaios Koumaras, Maria L. Garcia Osma, Daniele Munaretto, Eneko Atxutegi, Jara Suárez de Puga, Ozgu Alay, Anna Brunstrom, and Anne Marie Cristina Bosneag. 2018. 5GENESIS: The Genesis of a flexible 5G Facility. In 2018 IEEE 23rd International Workshop on Computer Aided Modeling and Design of Communication Links and Networks (CAMAD). 1-6. https://doi.org/10.1109/CAMAD.2018.8514956

[13] Michail-Alexandros Kourtis, Begona Blanco, Jordi Pérez-Romero, Dimitris Makris, Michael J. Mcgrath, George Xilouris, Daniele Munaretto, Rubén Solozabal, Aitor 
Sanchoyerto, Ioannis Giannoulakis, Emmanouil Kafetzakis, Vincenzo Riccobene, Elisa Jimeno, Anastasios Kourtis, Ramon Ferrús, Fidel Liberal, Harilaos Koumaras, Alexandros Kostopoulos, and Ioannis Chochliouros. 2019. A Cloud-Enabled Small Cell Architecture in 5G Networks for Broadcast/Multicast Services. IEEE Transactions on Broadcasting 65, 2 (2019), 414-424. https://doi.org/10.1109/TBC. 2019.2901394

[14] Kashif Mahmood, Pål Grønsund, Anastasius Gavras, Maria Barros Weiss, Dan Warren, Christos Tranoris, Andrea F. Cattoni, and Paul Muschamp. 2019. Design of $5 \mathrm{G}$ End-to-End Facility for Performance Evaluation and Use Case Trials. In 2019 IEEE 2nd 5G World Forum (5GWF). 341-346. https://doi.org/10.1109/5GWF 2019.8911639
[15] Cise Midoglu, Ȯzgü Alay, and Carsten Griwodz. 2019. Evaluation Framework for Real-Time Adaptive 360-Degree Video Streaming over 5G Networks. In Proceedings of the 2019 on Wireless of the Students, by the Students, and for the Students Workshop (S3'19). Association for Computing Machinery, New York, NY, USA, 6-8. https://doi.org/10.1145/3349621.3355726

[16] Almudena Diaz Zayas, Andrea F. Cattoni, Carlos Cardenas, Oscar Castañeda, Janie Baños, Pedro Merino, and Michael Dieudonne. 2018. TRIANGLE: a Platform to Validate 5G KPIs in End to End scenarios. In 2018 IEEE 23rd International Workshop on Computer Aided Modeling and Design of Communication Links and Networks (CAMAD). 1-7. https://doi.org/10.1109/CAMAD.2018.8514947 